\title{
The expression profiling of microRNA in systemic sclerosis-associated pulmonary arterial hypertension
}

\author{
Yu-Xia Huang ${ }^{1 \#}$, Fei Li ${ }^{2 \#}$, Dong Liu ${ }^{3 \#}$, Yuan-Yuan Sun ${ }^{1}$, Qin-Hua Zhao ${ }^{1}$, Rong Jiang ${ }^{1}$, Lan Wang ${ }^{1}$, \\ Ping Yuan ${ }^{1}$, Jin-Ming Liu' ${ }^{1}$, Yue $\mathrm{Wu}^{4}$, Ji Zhang ${ }^{3}$ \\ ${ }^{1}$ Department of Cardio-Pulmonary Circulation, Shanghai Pulmonary Hospital, School of Medicine, Tongji University, Shanghai, China; \\ ${ }^{2}$ Department of Radiology, Shanghai Pulmonary Hospital, School of Medicine, Tongji University, Shanghai, China; ${ }^{3}$ Department of Wuxi Lung \\ Transplant Center, Wuxi People's Hospital Affiliated to Nanjing Medical University, Wuxi, China; ${ }^{4}$ Department of Clinical Laboratory, Shanghai \\ Pulmonary Hospital, School of Medicine, Tongji University, Shanghai, China \\ Contributions: (I) Conception and design: J Zhang, JM Liu, Y Wu; (II) Administrative support: None; (III) Provision of study materials or patients: \\ None; (IV) Collection and assembly of data: YX Huang, F Li, D Liu, R Jiang, YY Sun, L Wang, JM Liu, Y Wu, P Yuan; (V) Data analysis and \\ interpretation: YX Huang, Y Wu, JM Liu, P Yuan, J Zhang; (VI) Manuscript writing: All authors; (VII) Final approval of manuscript: All authors. \\ "These authors contributed equally to this work. \\ Correspondence to: Dr. Jin-Ming Liu, MD. Department of Cardio-Pulmonary Circulation, Shanghai Pulmonary Hospital, School of Medicine, \\ Tongji University, No. 507 Zhengmin Road, Shanghai 200433, China. Email: jinmingliu@tongji.edu.cn; Dr. Yue Wu, MD. Department of \\ Clinical Laboratory, Shanghai Pulmonary Hospital, School of Medicine, Tongji University, No. 507 Zhengmin Road, Shanghai 200433, China. \\ Email: wuyue7721@163.com; Dr. Ji Zhang, MD. Department of Wuxi Lung Transplant Center, Wuxi People's Hospital Affiliated to Nanjing \\ Medical University, 299 Qingyang Road, Wuxi 214023, China. Email: sophie78@yeah.net.
}

Background: The role of microRNAs (miRNAs) in the pathogenesis of systemic sclerosis-associated pulmonary arterial hypertension (SSc-PAH) remains to be fully elucidated. This study evaluated the expression profile of miRNAs in the lung tissue of patients with SSc-PAH.

Methods: Lung tissue samples were collected from 3 SSc-PAH patients and 4 healthy controls. A small RNA high throughput sequence approach was used for screening the differentially expressed miRNAs in the lung tissue samples. Real-time quantitative reverse transcription polymerase chain reaction (qRT-PCR) was used to validate 4 highly significant differentially expressed miRNAs. Gene Ontology and KEGG (Kyoto Encyclopedia of Genes and Genomes) enrichment analysis for mRNAs were performed using the R package clusterProfiler software.

Results: A total of 82 upregulated miRNAs and 35 downregulated miRNAs were detected in the lung tissues of patients with SSc-PAH compared with healthy controls. GO enrichment analysis demonstrated that the upregulated target genes were closely involved in biological processes such as nervous system development, anatomical structure morphogenesis, system development, cellular macromolecule metabolic processes, and cellular processes. The downregulated target genes were involved in the plasma membrane bound cell projection morphogenesis and the regulation of macromolecule metabolic processes. The KEGG enrichment analysis showed that the upregulated genes were associated with important pathways involved in cancer biology, and the target genes of the downregulated miRNAs were involved in axon guidance. High throughput sequencing and qRT-PCR revealed that hsa-miR-205-5p and hsa-miR-539-3p were differentially expressed in SSc-PAH tissue. The target genes of hsa-miR-205-5p and hsa-miR-539-3p, IRF1and ADCYAP1, respectively, were verified using the high throughput dataset GSE48149.

Conclusions: miRNAs may play an important role in the pathogenesis of SSc-PAH, and hsa-miR-205-5p and hsa-miR-539-3p may be potential therapeutic targets in patients with SSc-PAH.

Keywords: Systemic sclerosis-associated pulmonary arterial hypertension (SSc-PAH); differentially expressed miRNAs; high throughput sequence 
Submitted Jul 26, 2021. Accepted for publication Sep 10, 2021.

doi: $10.21037 / \mathrm{atm}-21-4342$

View this article at: https://dx.doi.org/10.21037/atm-21-4342

\section{Introduction}

Systemic sclerosis (SSc) is an autoimmune connective tissue disease (CTD) characterized by small-vessel vasculopathy, inflammation, and immunodysfunction (1). Pulmonary arterial hypertension (PAH) associated with CTD accounts for about $15-25 \%$ of all $\mathrm{PAH}$, and SSc-associated $\mathrm{PAH}$ is the most common type of CTD-PAH (2). The prevalence of $\mathrm{PAH}$ in SSc patients ranges from $5-12 \%$ and is the leading cause of death in these patients, accounting for approximately $15 \%$ of SSc-related deaths $(3,4)$. Despite advances in targeted treatment regimens, the survival of SSc-PAH patients remains poor (5), with a mortality rate of about $50 \%$ at 3 years $(6,7)$. Recently, it was reported that serum uric acid was a risk factor and associated with the disease severity of the SSc-PAH (8). Furthermore, age, sex, mixed venous oxygen saturation, and WHO functional class were associated with the prognosis of the SSc-PAH (9). In addition to these known parameters, we need to find and understand more underlying risk factors.

SSc-PAH is characterized by abnormal vascular proliferation and remodeling of the small- to mediumsized pulmonary vasculature $(3,10)$. MicroRNAs (miRNAs) are small, non-coding RNA molecules with a size of approximately 22 nucleotides. They are involved in posttranscriptional negative regulation of genes by binding the partially complementary sequences of the target mRNA $(11,12)$. Studies have shown that miRNAs played an important role in the development and regulation of vascular and fibroproliferative alterations associated with SSc pathogenesis $(13,14)$. It has been reported that the dysregulation of miRNAs, including hsa-miR-193b, hsamiR-21, hsa-miR-31, hsa-miR-155, and hsa-miR-196a, can contribute to the proliferative vasculopathy of SSc $(15-17)$. One previous study showed that the expression levels of plasma miR-20a-5p was lower in SSc-PAH patients, and plasma miR-20a-5p and miR-203a-3p were linked with the occurrence of SSc-PAH in female patients with anti-centromere antibody-positive limited cutaneous SSc (18). Additionally, another study have indicated that miRNAs may play a vital role in the process of vascular inflammation (19), which is believed to be a major cause of poor prognosis in patients with SSc-PAH. However, the precise role of miRNAs in the development of SSc$\mathrm{PAH}$ has not been fully elucidated and there is a paucity of high throughput studies investigating the expression of miRNAs in the lung tissue of SSc-PAH patients.

This current study used a high throughput approach for screening differentially expressed miRNAs in the lung tissue of SSc-PAH patients and identified their possible roles in the pathogenesis of SSc-PAH. We present the following article in accordance with the MDAR reporting checklist (available at https://dx.doi.org/10.21037/atm-21-4342).

\section{Methods}

\section{Study population}

Lung tissue samples were obtained from SSc-PAH patients $(n=3)$ and healthy donors $(n=4)$ undergoing thoracic surgery for lung transplantation at the Shanghai Pulmonary Hospital and Wuxi People's Hospital, China.

Patients were included in the study if they: (I) satisfied the 2017 American College of Rheumatology/European League against Rheumatism (ACR/EULAR) criteria for the classification of SSc (14); and (II) were diagnosed with $\mathrm{PAH}$ by right heart catheterization (RHC) measured at rest, with mean pulmonary arterial pressure $(\mathrm{mPAP}) \geq 25 \mathrm{mmHg}$, pulmonary arterial wedge pressure (PAWP) $\leq 15 \mathrm{mmHg}$, and pulmonary vascular resistance (PVR) $\geq 3 \mathrm{WU}$ based on the 2015 European Society of Cardiology (ESC) and the European Respiratory Society (ERS) guidelines (20). Patients were excluded if they presented with the following: (I) SSc complicated with other syndromes, such as SSc with systemic lupus erythematosus or SSc with Sjogren's syndrome; (II) SSc with post-capillary pulmonary hypertension (PH); (III) chest radiograph with significant parenchymal lung disease; and (IV) echocardiography with evidence of left heart disease.

This study was approved by the institutional review board of the Shanghai Pulmonary Hospital (K20-195Y) and Wuxi People's Hospital (2020-492). All participants provided written informed consent and indicated a willingness to donate their tissue samples for research. All procedures followed were in accordance with the ethical standards of the responsible committee on human experimentation (institutional and national) and with the 
Helsinki Declaration (as revised in 2013).

\section{High throughput sequencing}

High throughput sequencing was performed by Oebiotech Biotechnology Co. (Shanghai, China). The miRNA raw data was reduced to cleaned sequences by removing lowquality reads and the Q20, Q30, and GC content of the raw data were calculated. Taken together, all the downstream analyses were based on the clean data. Small RNA sequencing procedures and analyses were performed by OEbiotech (Shanghai, China). For preliminary analysis, we determined the length distribution of the clean sequence in the reference genome.

To identify and remove the ribosomal RNAs (rRNAs), small nuclear RNAs (snRNAs), transfer RNAs (tRNAs), and small nucleolar RNAs (snoRNAs), the clean reads were aligned with the small RNAs from the Rfam database (v2.2.28+) and GenBank database (http://www. ncbi.nlm.nih.gov/) using BLAST (v2.2.28+). Known miRNAs were identified by comparing with the miRBase (v21) database (http://www.mirbase.org/) and the known miRNA expression patterns in different samples were analyzed.

\section{Identification and functional enrichment analysis of differentially expressed miRNAs}

The miRNAs with $\mathrm{P}<0.05$ and $\mid \log 2$ foldchange $\mid>1$ were screened as differentially expressed miRNAs. Target genes of differentially expressed miRNAs were predicted using 3 online miRNAs target prediction tools, including Targetscan, miRDB, DIANA-microT-CDS.

To further understand the functional categories and related gene functions, such as metabolic pathways or signal transduction pathways, of the differentially expressed miRNAs, Gene Ontology (GO) analysis and Kyoto Encyclopedia of Genes and Genomes (KEGG) analysis of the differentially expressed miRNAs was performed using the clusterProfiler in the statistical programming language $\mathrm{R}$ (http://www.R-project.org). An adjusted false discovery rate (FDR) $\mathrm{P}<0.05$ was considered statistically significant. The top 10 significantly GO and KEGG pathways were selected.

\section{$R N A$ isolation and real-time quantitative reverse transcription polymerase chain reaction ( $q R T-P C R)$}

Lung tissue samples were obtained from SSc-PAH lung transplant patients and healthy donors and stored at $-80{ }^{\circ} \mathrm{C}$ until use. Total RNA was extracted from lung tissue samples using the miRNeasy mini kit (Qiagen, Hilden, Germany). RNA purity was calculated using the NanoDrop 2000 spectrophotometer (Thermo Scientific, USA) at 260/280 $\mathrm{nm}$ (ratio 1.9:2.1).

The highly significant differentially expressed miRNAs were selected based on the stricter criteria of q-value $<0.1$ and $\mid \log 2$ foldchange $\mid>3$. The expression of the highly significant differentially expressed miRNAs was validated by qRT-PCR. The extracted total RNA $(1 \mu \mathrm{g})$ was reversely transcribed into cDNA using the ReverTra Ace qPCR RT Kit (TOYOBO FSQ101, Japan) and qRTPCR was performed with the SYBR Green PCR master mix (TOYOBO, QPK201, Osaka, Japan) according to the manufacturer's instructions. U6 was used for qRTPCR normalization. The primer pairs used are listed in Table 1. All real-time PCR reactions were carried out in duplicates and all data were calculated with the $2^{-\Delta \Delta C t}$ equation.

\section{Verification of differentially expressed $m R N A s$ in the SSc-PAH samples based on the GEO database}

The mRNAs profile GSE48149 was obtained from the Gene Expression Omnibus (GEO) public database and analyzed. The microarray dataset contained lung tissue samples from 9 healthy controls and 10 SSc-PAH patients. The microarray data from the GSE48149 dataset was downloaded and analyzed using the online web tool GEO2R. The mRNAs in the microarray with a $\mathrm{P}<0.05$ and $\mid \log 2$ foldchange $\mid>1$ were considered differentially expressed mRNAs.

\section{Statistic methods}

Continuous variables with normal distribution were expressed as mean \pm standard deviation (SD), and nonnormal variables were expressed as median (interquartile range). Categorical variables were expressed as numbers (percentage). The identification of differentially expressed miRNAs between SSc-PAH and healthy control tissue samples was performed using the Student's $t$-test and the limma package in the R statistical software. All GO and KEGG analyses, and other computational procedures were carried out using in-house programs written in $\mathrm{R}$ and Graph Prism 9. 
Table 1 Primers for the differentially expressed miRNAs used in stem-loop qRT-PCR

\begin{tabular}{|c|c|c|}
\hline miRNAs & Orientation & Sequences \\
\hline hsa-miR-205-5p & Reverse & ACACTCCAGCTGGG TCCTTCATTCCAC \\
\hline \multirow[t]{2}{*}{ hsa-miR-31-5p } & Forward & CAGTGCGTGTCGTGGAGT \\
\hline & Reverse & ACACTCCAGCTGGG AGGCAAGATGCTG \\
\hline hsa-miR-6510-3p & Reverse & ACACTCCAGCTGGG CACCGACTCTGTCT \\
\hline \multirow[t]{2}{*}{ hsa-miR-31-3p } & Forward & CAGTGCGTGTCGTGGAGT \\
\hline & Reverse & ACACTCCAGCTGGG TGCTATGCCAACAT \\
\hline hsa-miR-376a-3p & Forward & CAGTGCGTGTCGTGGAGT \\
\hline hsa-miR-514a-3p & Reverse & ACACTCCAGCTGGG ATTGACACTTCTGT \\
\hline \multirow[t]{2}{*}{ hsa-miR-539-3p } & Forward & CAGTGCGTGTCGTGGAGT \\
\hline & Reverse & ACACTCCAGCTGGGATCATACAAGGACA \\
\hline \multirow[t]{2}{*}{ hsa-miR-154-3p } & Forward & CAGTGCGTGTCGTGGAGT \\
\hline & Reverse & ACACTCCAGCTGGGAATCATACACGGTTG \\
\hline \multirow[t]{2}{*}{ U6 } & Forward & CTCGCTTCGGCAGCACA \\
\hline & Reverse & AACGCTTCACGAATTTGCGT \\
\hline
\end{tabular}

qRT-PCR, quantitative reverse transcription polymerase chain reaction.

Table 2 The clinical characteristic of 3 patients with systemic sclerosis-associated pulmonary arterial hypertension

\begin{tabular}{lc}
\hline Characteristics & Mean [range] or No. (\%) \\
\hline Mean age (years) & $54.5[52-65]$ \\
Mean PAP* $(\mathrm{mmHg})$ & $61.3[56-64]$ \\
Gender & $3(100.0)$ \\
Male & \\
Co-existing conditions & $3(100.0)$ \\
SSc-PF & $1(33.3)$ \\
Diabetes & \\
Treatment & $3(100.0)$ \\
Systematic glucocorticoid & $2(66.7)$ \\
Cyclophosphamide &
\end{tabular}

*, pulmonary artery pressure measured by echocardiography. PAP, pulmonary artery pressure; SSc-PF, systemic sclerosisassociated pulmonary fibrosis.

\section{Results}

\section{Clinical characteristics of the SSc-PAH patients}

A total of 3 SSc-PAH patients were enrolled in the study and all patients were male, with a mean age of 54.5 years. The clinical characteristics of the patients are listed in Table 2.

\section{Analysis of the differentially expressed miRNAs}

The results of the chip detection revealed 117 differentially expressed miRNAs that satisfied the criteria of $\mathrm{P}<0.05$ and $\mid \log 2$ foldchange $\mid>1$. The distribution of miRNA regulation is shown in the volcano plot in Figure 1. A total of 82 miRNAs were upregulated and 35 were downregulated. A heatmap was constructed based on the differentially expressed miRNAs between the SSc-PAH patients and the healthy controls by using unsupervised 


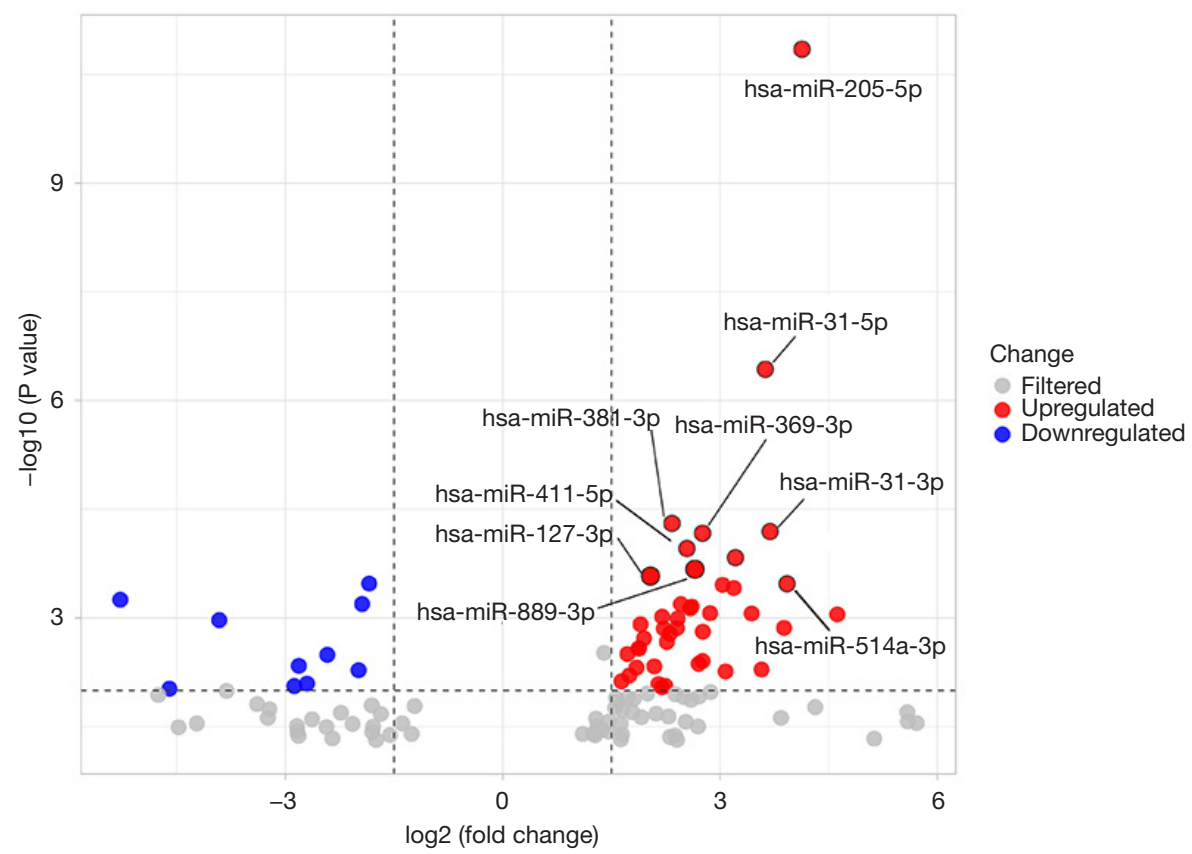

Figure 1 Volcano plot highlighting the significant genes. The $y$-axis corresponds to negative $\log 10$ (adjusted $\mathrm{P}$ ), and the $\mathrm{x}$-axis displays the $\log 2$ foldchange value. The red dots represent the upregulated differentially expressed genes $(\mathrm{P}<0.05, \log 2$ foldchange $>1)$ between normal and SSc-PAH lung tissues. The blue dots represent the downregulated genes $(\mathrm{P}<0.05, \log 2$ foldchange $<-1)$. SSc-PAH, systemic sclerosisassociated pulmonary arterial hypertension.

hierarchical clustering analysis (Figure 2). The highly significant differentially expressed miRNAs are listed in Table 3. The q-value of the 4 miRNAs: miR-205-5p, miR6510-3p, miR-31-5p, and miR-31-3p were less than 0.05 .

\section{GO enrichment analysis of target genes of differently expressed miRNAs}

For upregulated miRNAs, GO annotations indicated that predicted target genes were closely related to biological processes, including nervous system development, anatomical structure morphogenesis, system development, cellular macromolecule metabolic processes, and cellular processes (Figure 3A). The results of the KEGG pathway enrichment analysis indicated that the important pathways were involved in cancer biology, which suggested that SSc$\mathrm{PAH}$ was a cancer-like disease (Figure $3 B$ ).

For the downregulated miRNAs, GO analysis showed that target genes were closely related to axon guidance, neuron projection guidance, cell morphogenesis involved in neuron differentiation, and anatomical structure morphogenesis (Figure 3C). KEGG analysis suggested that the significant pathways were involved in axon guidance
(Figure 3D).

\section{qRT-PCR analysis for bighly significant differentially expressed miRNAs}

The qRT-PCR validation showed that the expression of hsa-miR-205-5p and hsa-miR-539-3p were significantly elevated in the tissue samples of SSc-PAH patients compared with healthy donors $(\mathrm{P}=0.00167$ and $\mathrm{P}<0.001$, respectively; Figure 4).

\section{Identification of the candidate genes of hsa-miR-205-5p and hsa-miR-539-3p}

The target genes of hsa-miR-205-5p and hsa-539-3p were predicted using 3 online prediction tools, including miRDB, Targetscan, and DIANA-microT-CDs (Figure 5). The differentially expressed mRNAs in the dataset GSE48149 were used to verify the targets genes of hsa-miR-205-5p and hsa-miR-539-3p. A total of 68 differentially downregulated genes were identified in the validation dataset GSE48149. Interferon regulatory factor 1 (IRF1) and adenylate cyclase activating polypeptide 1 (ADCYAP1) were identified as 


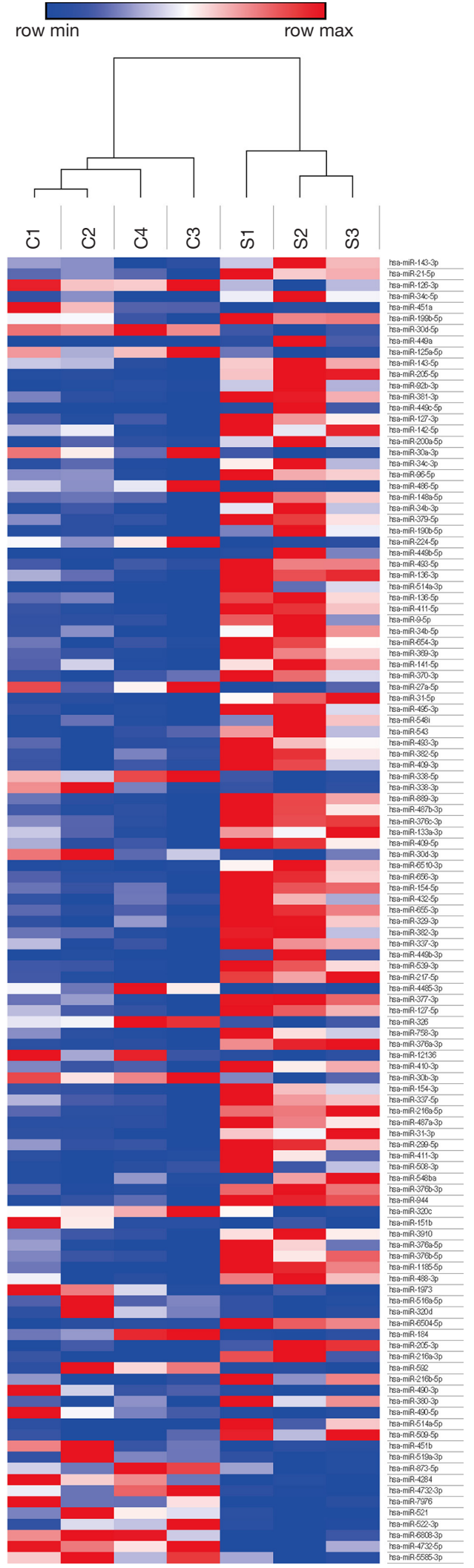

Figure 2 Heatmap of all the differentially expressed miRNAs. the target genes of hsa-miR-205-5p and hsa-miR-539-3p, respectively (Figure 6).

\section{Discussion}

Though with the advanced therapies, the prognosis of SSc-PAH was still poor. Recently, more and more studies have used the multi-omics analysis to identify the differently miRNAs and mRNAs in the serum and lung tissue of SSc-PAH, however, there was still lacking the specific biomarkers for SSc-PAH, the studies related to the miRNAs in the lung tissue of SSc-PAH was still limited. To our knowledge, this is the first study to use high throughput sequencing to examine the miRNA expression profile in the lung tissue of SSc-PAH patients. The present study identified hsa-miR-205-5p and hsa-miR-539-3p as differentially expressed miRNAs in the lung tissue of SScPAH patients, which might help us understand the roles of miRNAs in the pathogenesis of SSc-PAH. Previous studies also indicated that the abnormal hsa-miR-205-5p and hsamiR-539-3p expressions existed in the various cancers $(21,22)$. These results demonstrated that the two miRNAs may be the potential biomarkers of the early diagnosis, disease evaluation and prognosis judgment in SSc-PAH.

The GO analysis results showed that both the up- and downregulated genes are involved in cellular processes, suggesting that dysfunction in cellular processes may be critical in the development of SSc-PAH. There is evidence that endothelial to mesenchyme transition is a cellular process that contributes to the pathogenesis of PAH (23). Moreover, during PAH development, cells in the pulmonary vascular wall become hyperproliferative which leads to cell proliferation and an increased remodeling of the pulmonary vasculature (24). In addition, the common GO term in both up- and downregulated miRNAs was anatomical structure morphogenesis. KEGG analysis showed that the target genes of upregulated and downregulated miRNAs are both closely associated with the axon guidance pathway. Furthermore, the significant pathways of the target genes of the upregulated miRNAs are linked to cancer biology. The upregulated miRNAs are also linked to epidermal growth factor receptor (EGFR) tyrosine kinase inhibitor resistance, Forkhead box $\mathrm{O}$ isoforms (FoxO) signaling pathway, and signaling pathways regulating pluripotency of stem cells. FoxOs are reportedly involved in vascular structural maintenance (25-27) and FoxO1 has been shown to be critical to the hyperproliferation of pulmonary artery smooth muscle 
Table 3 The highly significant differentially expressed miRNAs

\begin{tabular}{lcccc}
\hline miRNA & log2foldchange & $P$ & $Q$ & Regulation \\
\hline hsa-miR-205-5p & 4.13 & 0.0000 & 0.0000 & Up \\
hsa-miR-31-5p & 3.62 & 0.0000 & 0.0004 & Up \\
hsa-miR-6510-3p & 4.13 & 0.0000 & 0.0004 & Up \\
hsa-miR-31-3p & 3.69 & 0.0001 & 0.0259 & Up \\
hsa-miR-376a-3p & 3.21 & 0.0001 & 0.0413 & Up \\
hsa-miR-514a-3p & 3.93 & 0.0003 & 0.0636 & Up \\
hsa-miR-539-3p & 3.04 & 0.0003 & 0.0636 & Up \\
hsa-miR-154-3p & 3.19 & 0.0004 & 0.0655 & Up \\
\hline
\end{tabular}

A

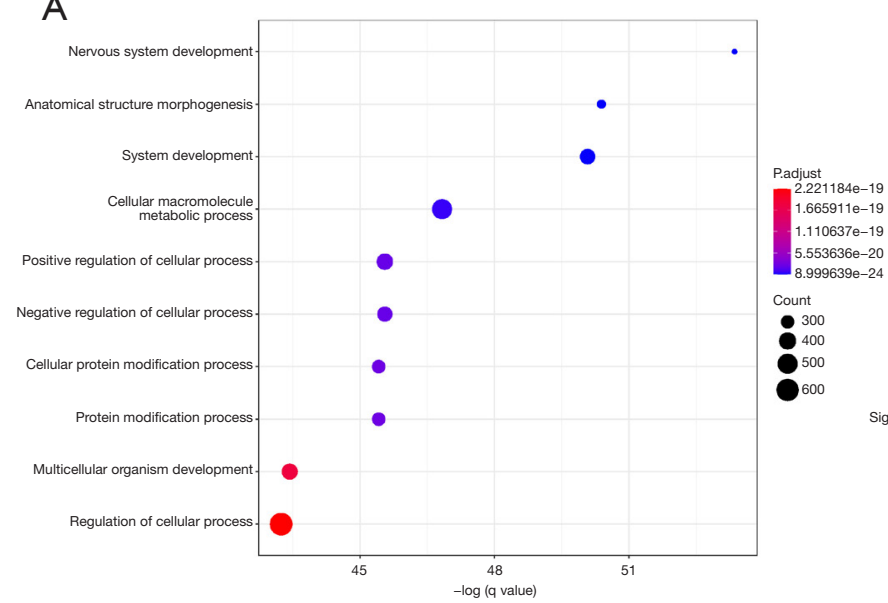

C

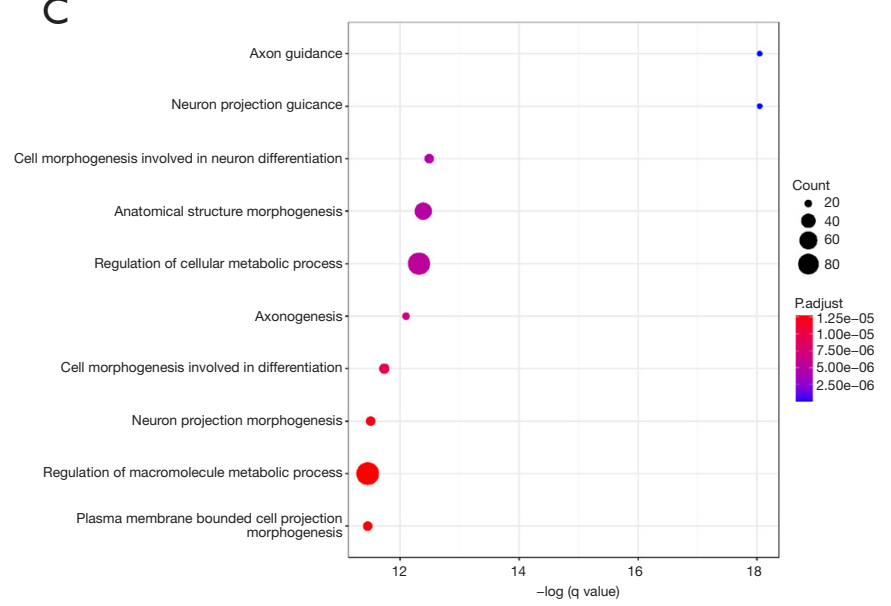

B

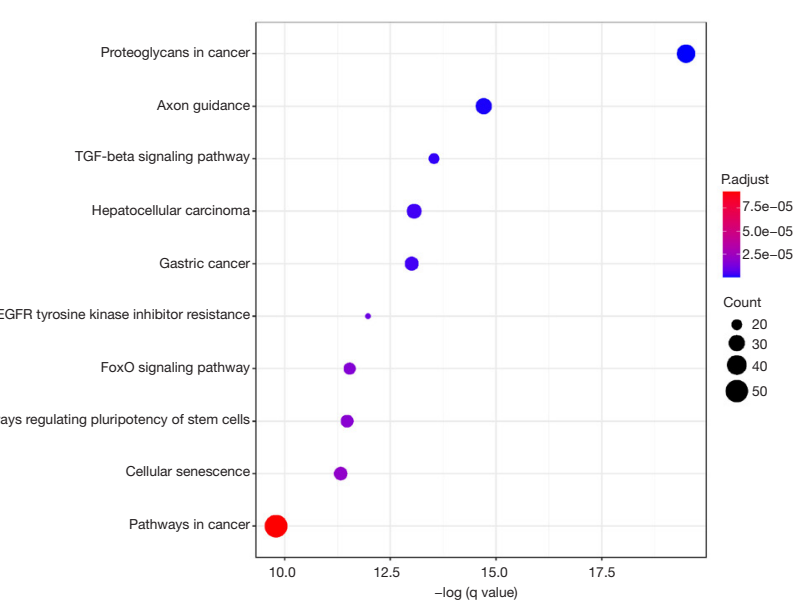

D

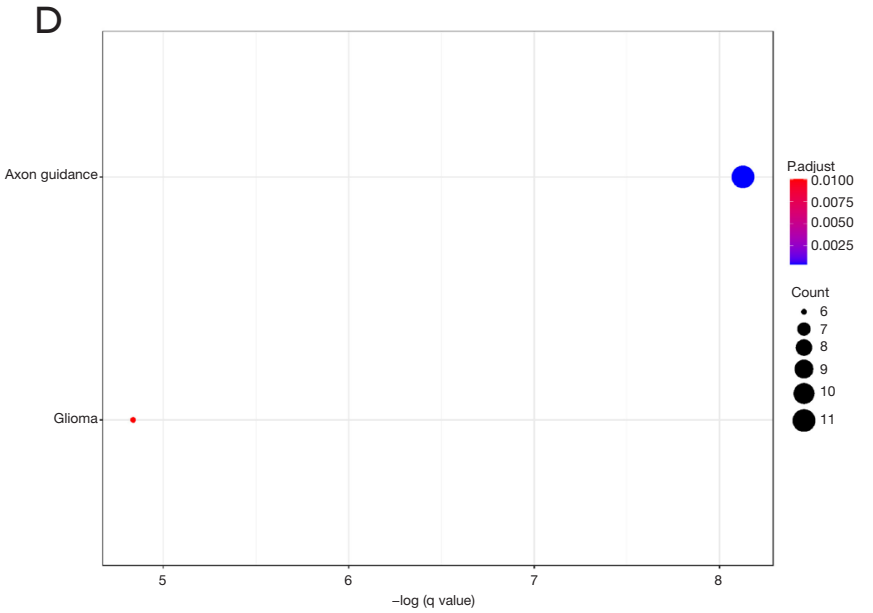

Figure 3 GO and KEGG enrichment analysis of differently expressed miRNA. (A) The top10 enriched GO terms for the predicted target genes of upregulated differentially expressed miRNAs. FDR corrected $\mathrm{P}<0.05$ was used as a threshold to select significant GO pathways. (B) The top 10 enriched KEGG terms for the predicted target genes of upregulated differentially expressed miRNAs. FDR corrected $\mathrm{P}<0.05$ was used as a threshold to select significant GO pathways. (C) The top 10 enriched GO terms for the predicted target genes of downregulated differentially expressed miRNAs. FDR corrected $\mathrm{P}<0.05$ was used as a threshold to select significant GO pathways. (D) The significant KEGG pathways related to the predicted target genes of downregulated differentially expressed miRNAs. FDR corrected $\mathrm{P}<0.05$ was used as a threshold to select significant GO pathways. GO, Gene Ontology; KEGG, Kyoto Encyclopedia of Genes and Genomes; FDR, false discovery rate. 
A

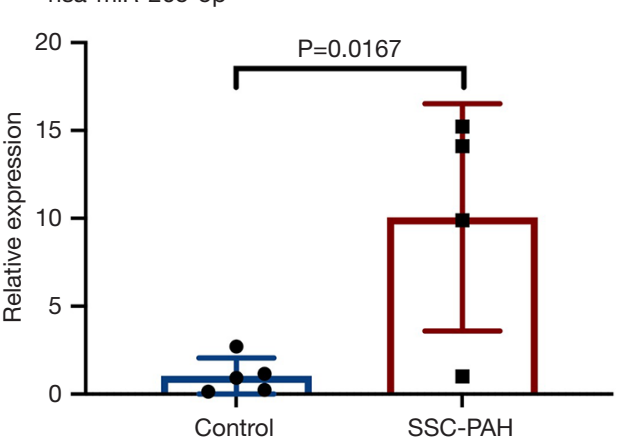

B

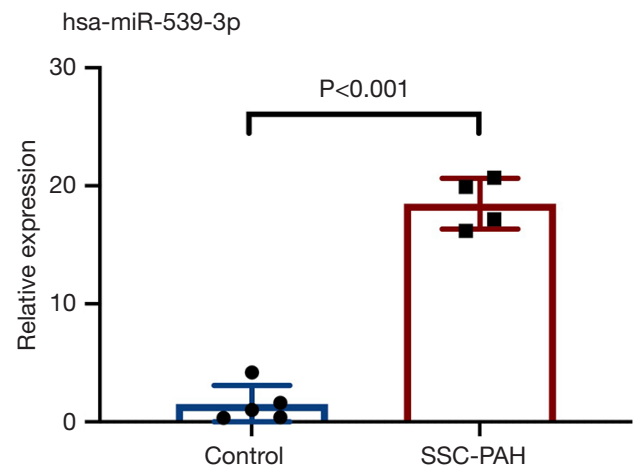

Figure 4 Real-time qRT-PCR verification results of hsa-miR-205-5p and hsa-miR-539-3p in 4 patients with SSc-PAH and 5 healthy controls. (A) The expression of hsa-miR-205-5p verified by real-time qRT-PCR. (B) The expression of hsa-miR-539-3p verified by real-time qRT-PCR. $\mathrm{P}<0.05$ was considered statistically significant. SSc-PAH, systemic sclerosis-associated pulmonary arterial hypertension; qRTPCR, quantitative reverse transcription polymerase chain reaction.

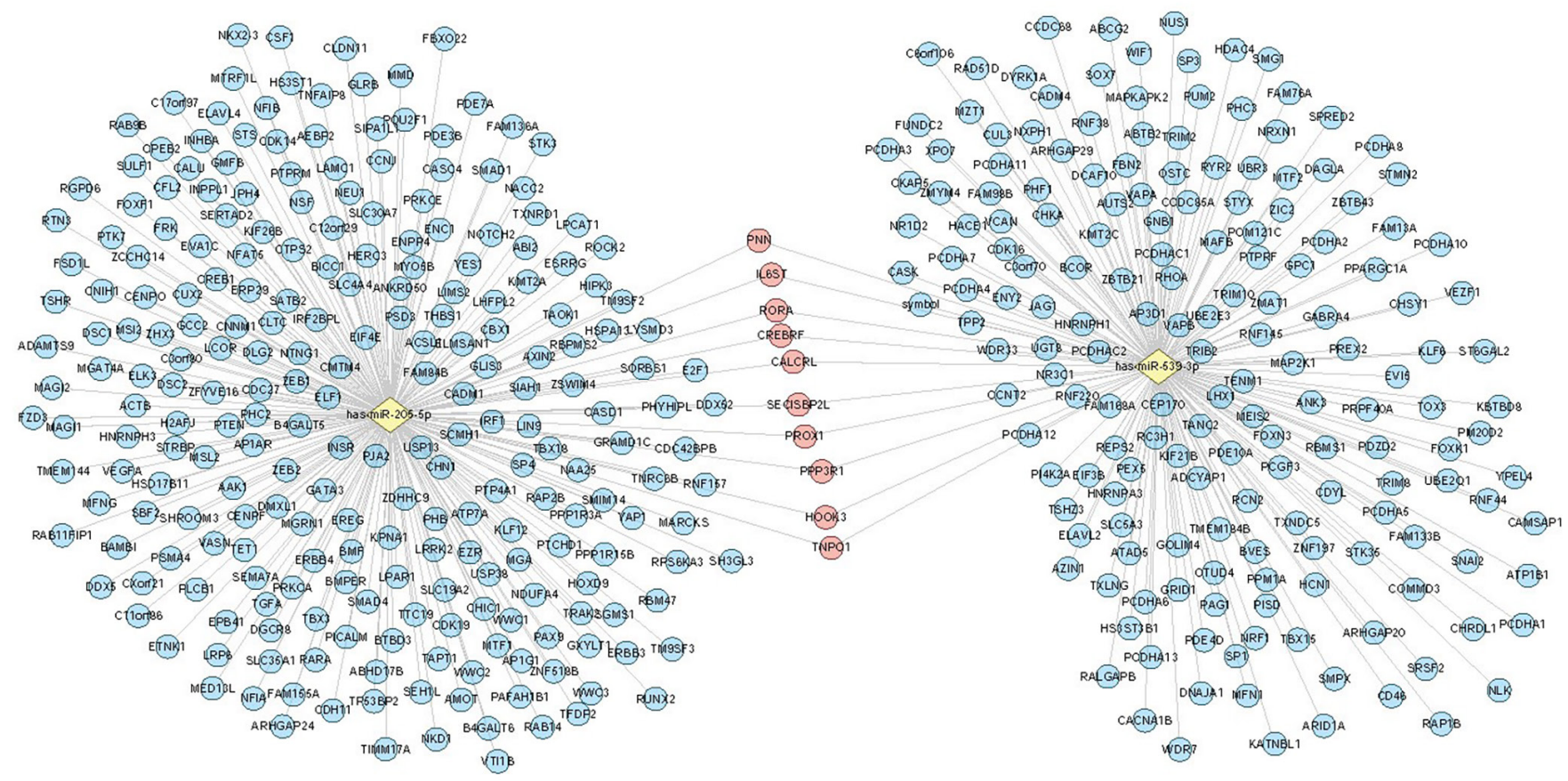

Figure 5 The predicted target genes of hsa-miR-205-5p and hsa-miR-539-3p.

cells (PASMCs) in pulmonary hypertension (27).

Prior studies by Gulei et al. demonstrated that upregulation of hsa-miR-205-5p inhibited epithelial to mesenchymal transition in colon cancer cells (28), suggesting that hsa-miR-205-5p plays a vital role in the development of cancer. In addition, hsa-miR-205-5p may play an important role in the development of SSc-PAH.
Studies have suggested that the dysregulation of miRNAs plays important roles in the development of pulmonary hypertension (29). In fact, hsa-miR-205-5p has been shown to be closely associated with the proliferation of PASMCs in PAH. Furthermore, hsa-miR-205-5p can inhibit smooth muscle cell proliferation by activating the ERK1/2 signaling pathway, and thus, may be a potential therapeutic target 
A

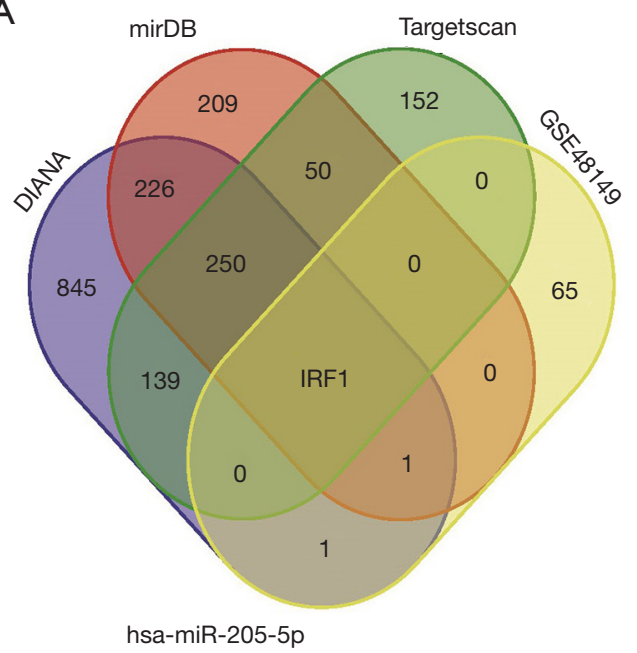

B

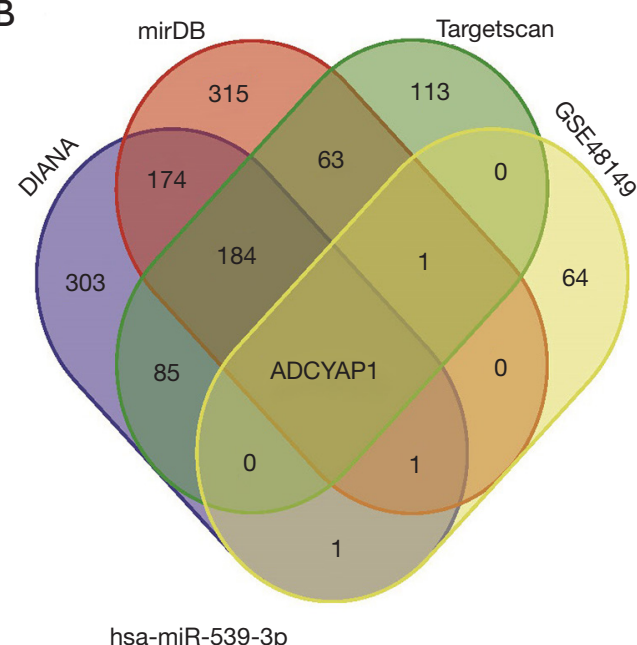

Figure 6 The venn diagram analysis of tagrget genes of hsa-miR-205-5p and hsa-miR-539-3p and differently epressed mRNAs in the dataset GSE48149. (A) The venn diagram showed the overlap of the target genes of hsa-miR-205-5p miRNAs predicted by three online tools and the differently expressed mRNAs in the dataset GSE48149 was IRF1. (B) The venn diagram showed the overlap of the target genes of hsa-miR-539-3p predicted by three online tools and in the dataset GSE48149 was ADCYAP1.

for PAH treatment (30). Tao et al. suggested that hsa-miR205-5p was downregulated in mice PASMCs under hypoxia conditions, and inhibited the cell proliferation through regulating ERK1/2 pathway (31). The similar mechanism was also observed in cancer, which might lead to PASMC cancer-like transformation (32). However, to date, there have been few studies examining the expression and role of hsa-miR-205-5p and hsa-miR-539-3p in SSc-PAH.

GO analysis results indicated that the target genes of hsamiR-205-5p were involved in all GO categories, especially in biological processes, including tube morphogenesis, negative regulation of development processes, and regulation of anatomical structure morphogenesis. This suggested that cellular processes and organ system development play vital roles in the development of SSc-PAH. Reports have suggested that endothelial to mesenchyme transition contributes significantly to the pathogenesis of PAH (20). Moreover, during PAH development, cells in the pulmonary vascular wall become hyperproliferative which leads to cell proliferation and an increase in remodeling of the pulmonary vasculature (24).

This study identified IRF1 and ADCYAP 1 as the target genes of the hsa-miR-205-5p and hsa-miR-539-3p, respectively. IRF1 has been reported to be associated with cell growth control and induction of apoptosis (32). Lee et al. showed that IRF1 inhibited the proliferation and migration of endothelial cells (33). Besides, IRF1 regulated the immune cell development (34,35). Both ADCYAP1 and IRF1 have been shown to exert antiproliferative effects in cells of various diseases (36,37). ADCYAP1 was a multifunctional neuropeptide with anti-inflammatory and cardioprotective functions (38). ADCYAP1 could indirectly vasodilate vessel through stimulating NO releasing (39). Therefore, hsa-miR-205-5p and hsa-miR-539-3p may target the two target genes to participate in the process of disease development by involving in the regulation of perivascular inflammatory cell function. Future studies are warranted to further elucidate the roles of IRF1 and ADCYAP1 in SSc-PAH.

\section{Study limitations}

There were several limitations to this study. First, there were few tissue samples examined this study, and this may lead to inadequate statistical power. Second, additional subgroup analysis of the gene expression of SSc patients and SSc-PAH patients may lead to further understanding of the development of SSc-PAH. Third, further experiments are required to verify the target genes of the differentially expressed miRNAs.

\section{Conclusions}

$\mathrm{PAH}$ is an incurable and common complication of 
SSc, that contributes to early mortality in SSc patients. Unfortunately, the genetic etiology of SSc-PAH has not been fully elucidated. Therefore, understanding the molecular mechanisms underlying the early development of SSc-PAH, in particular, the role of miRNAs, is crucial. This study demonstrated that the miRNAs hsa-miR-205-5p and hsa-miR-539-3p may play important roles in the pathogenesis of SSc-PAH.

\section{Acknowledgments}

Funding: This study was supported by the Program of National Natural Science Foundation of China (81870042 and 81900050), the Program of Natural Science Foundation of Shanghai (21ZR1453800 and 18ZR1431500), the Program of Shanghai Municipal Commission of Health (20204Y0382), the Youth Project of Shanghai Municipal Commission of Health and Family Planning (20174Y0143), and the Program of Shanghai Pulmonary Hospital (FKLY20005).

\section{Footnote}

Reporting Checklist: The authors have completed the MDAR reporting checklist. Available at https://dx.doi. org/10.21037/atm-21-4342

Data Sharing Statement: Available at https://dx.doi. org/10.21037/atm-21-4342

Conflicts of Interest: All authors have completed the ICMJE uniform disclosure form (available at https://dx.doi. org/10.21037/atm-21-4342). The authors have no conflicts of interest to declare.

Ethical Statement: All authors are accountable for all aspects of the work in ensuring that questions related to the accuracy or integrity of any part of the work are appropriately investigated and resolved. All procedures followed were in accordance with the ethical standards of the responsible committee on human experimentation (institutional and national) and with the Helsinki Declaration (as revised in 2013). Shanghai Pulmonary Hospital, Wuxi People's Hospital reviewed and approved the protocol form prior to initiation of the study (approval number: K20-195Y and 2020-492). All participants provided written informed consent and indicated a willingness to donate their tissue samples for research.
Open Access Statement: This is an Open Access article distributed in accordance with the Creative Commons Attribution-NonCommercial-NoDerivs 4.0 International License (CC BY-NC-ND 4.0), which permits the noncommercial replication and distribution of the article with the strict proviso that no changes or edits are made and the original work is properly cited (including links to both the formal publication through the relevant DOI and the license). See: https://creativecommons.org/licenses/by-nc-nd/4.0/.

\section{References}

1. Tabata K, Mikita N, Yasutake M, et al. Up-regulation of IGF-1, RANTES and VEGF in patients with anticentromere antibody-positive early/mild systemic sclerosis. Mod Rheumatol 2021;31:171-6.

2. Visovatti S, Lewis C, Sanderson R, et al. Beyond Scleroderma: Pulmonary Arterial Hypertension in Patients with Other Connective Tissue Diseases. In: Ford HJ, Heresi GA, Risbano MG. editors. Cham: Springer International Publishing, 2020:51-60.

3. Chaisson NF, Hassoun PM. Systemic sclerosisassociated pulmonary arterial hypertension. Chest 2013;144:1346-56.

4. Launay D, Sobanski V, Hachulla E, et al. Pulmonary hypertension in systemic sclerosis: different phenotypes. Eur Respir Rev 2017;26:170056.

5. Argula RG, Ward C, Feghali-Bostwick C. Therapeutic Challenges And Advances In The Management Of Systemic Sclerosis-Related Pulmonary Arterial Hypertension (SSc-PAH). Ther Clin Risk Manag 2019;15:1427-42.

6. Zheng JN, Li Y, Yan YM, et al. Identification and Validation of Key Genes Associated With Systemic Sclerosis-Related Pulmonary Hypertension. Front Genet 2020;11:816.

7. Le Pavec J, Humbert M, Mouthon L, et al. Systemic sclerosis-associated pulmonary arterial hypertension. Am J Respir Crit Care Med 2010;181:1285-93.

8. Simpson CE, Damico RL, Hummers L, et al. Serum uric acid as a marker of disease risk, severity, and survival in systemic sclerosis-related pulmonary arterial hypertension. Pulm Circ 2019;9:2045894019859477.

9. Condliffe R, Kiely DG, Peacock AJ, et al. Connective tissue disease-associated pulmonary arterial hypertension in the modern treatment era. Am J Respir Crit Care Med 2009;179:151-7.

10. Morrisroe K, Stevens W, Huq M, et al. Survival and 
quality of life in incident systemic sclerosis-related pulmonary arterial hypertension. Arthritis Res Ther 2017;19:122.

11. Matsuyama H, Suzuki HI. Systems and Synthetic microRNA Biology: From Biogenesis to Disease Pathogenesis. Int J Mol Sci 2019;21:132.

12. Brancati $G$, Großhans H. An interplay of miRNA abundance and target site architecture determines miRNA activity and specificity. Nucleic Acids Res 2018;46:3259-69.

13. Iizasa H, Kim H, Kartika AV, et al. Role of Viral and Host microRNAs in Immune Regulation of Epstein-Barr VirusAssociated Diseases. Front Immunol 2020;11:367.

14. Odler B, Foris V, Gungl A, et al. Biomarkers for Pulmonary Vascular Remodeling in Systemic Sclerosis: A Pathophysiological Approach. Front Physiol 2018;9:587.

15. Iwamoto N, Vettori S, Maurer B, et al. Downregulation of miR-193b in systemic sclerosis regulates the proliferative vasculopathy by urokinase-type plasminogen activator expression. Ann Rheum Dis 2016;75:303-10.

16. Tsai CY, Hsieh SC, Wu TH, et al. Pathogenic Roles of Autoantibodies and Aberrant Epigenetic Regulation of Immune and Connective Tissue Cells in the Tissue Fibrosis of Patients with Systemic Sclerosis. Int J Mol Sci 2020;21:3069.

17. Henry TW, Mendoza FA, Jimenez SA. Role of microRNA in the pathogenesis of systemic sclerosis tissue fibrosis and vasculopathy. Autoimmun Rev 2019;18:102396.

18. Wuttge DM, Carlsen AL, Teku G, et al. Circulating Plasma microRNAs In Systemic Sclerosis-Associated Pulmonary Arterial Hypertension. Rheumatology (Oxford) 2021. [Epub ahead of print]. doi: 10.1093/rheumatology/ keab300.

19. Reiff D, Crayne CB, Mannion ML, et al. Characteristics of coexisting localized scleroderma and inflammatory arthritis. Eur J Rheumatol 2019. [Epub ahead of print]. doi: 10.5152/eurjrheum.2019.19147.

20. Galiè N, Humbert M, Vachiery JL, et al. 2015 ESC/ERS Guidelines for the diagnosis and treatment of pulmonary hypertension: The Joint Task Force for the Diagnosis and Treatment of Pulmonary Hypertension of the European Society of Cardiology (ESC) and the European Respiratory Society (ERS): Endorsed by: Association for European Paediatric and Congenital Cardiology (AEPC), International Society for Heart and Lung Transplantation (ISHLT). Eur Heart J 2016;37:67-119.

21. Liu H, Yang M, Zhang Y, et al. The effect of miR539 regulating TRIAP1 on the apoptosis, proliferation, migration and invasion of osteosarcoma cells. Cancer Cell Int 2021;21:227.

22. Ghorbanmehr N, Gharbi S, Korsching E, et al. miR21-5p, miR-141-3p, and miR-205-5p levels in urinepromising biomarkers for the identification of prostate and bladder cancer. Prostate 2019;79:88-95.

23. Babicheva A, Yuan JX. Endothelial Notch1 in Pulmonary Hypertension. Circ Res 2019;124:176-9.

24. Cheng J, Wu Q, Lv R, et al. MicroRNA-449a Inhibition Protects H9C2 Cells Against Hypoxia/ReoxygenationInduced Injury by Targeting the Notch-1 Signaling Pathway. Cell Physiol Biochem 2018;46:2587-600.

25. Paik JH. FOXOs in the maintenance of vascular homoeostasis. Biochem Soc Trans 2006;34:731-4.

26. Wilhelm K, Happel K, Eelen G, et al. FOXO1 couples metabolic activity and growth state in the vascular endothelium. Nature 2016;529:216-20.

27. Pullamsetti SS, Perros F, Chelladurai P, et al. Transcription factors, transcriptional coregulators, and epigenetic modulation in the control of pulmonary vascular cell phenotype: therapeutic implications for pulmonary hypertension (2015 Grover Conference series). Pulm Circ 2016;6:448-64.

28. Gulei D, Magdo L, Jurj A, et al. The silent healer: miR205-5p up-regulation inhibits epithelial to mesenchymal transition in colon cancer cells by indirectly up-regulating E-cadherin expression. Cell Death Dis 2018;9:66.

29. Carregal-Romero S, Fadón L, Berra E, et al. MicroRNA Nanotherapeutics for Lung Targeting. Insights into Pulmonary Hypertension. Int J Mol Sci 2020;21:3253.

30. Feng L, Wei J, Liang S, et al. miR-205/IRAK2 signaling pathway is associated with urban airborne PM2.5-induced myocardial toxicity. Nanotoxicology 2020;14:1198-212.

31. Tao W, Sun W, Zhu H, et al. miR-205-5p suppresses pulmonary vascular smooth muscle cell proliferation by targeting MICAL2-mediated Erk1/2 signaling. Microvasc Res 2019;124:43-50.

32. Ferrari E, Gandellini P. Unveiling the ups and downs of miR-205 in physiology and cancer: transcriptional and post-transcriptional mechanisms. Cell Death Dis 2020;11:980.

33. Lee JH, Chun T, Park SY, et al. Interferon regulatory factor-1 (IRF-1) regulates VEGF-induced angiogenesis in HUVECs. Biochim Biophys Acta 2008;1783:1654-62.

34. Feng H, Zhang YB, Gui JF, et al. Interferon regulatory factor 1 (IRF1) and anti-pathogen innate immune responses. PLoS Pathog 2021;17:e1009220. 
35. Zhang XJ, Zhang P, Li H. Interferon regulatory factor signalings in cardiometabolic diseases. Hypertension 2015;66:222-47.

36. Romeo G, Fiorucci G, Chiantore MV, et al. IRF-1 as a negative regulator of cell proliferation. J Interferon Cytokine Res 2002;22:39-47.

37. Ravni A, Eiden LE, Vaudry H, et al. Cycloheximide treatment to identify components of the transitional transcriptome in PACAP-induced PC12 cell differentiation. J Neurochem 2006;98:1229-41.

Cite this article as: Huang YX, Li F, Liu D, Sun YY, Zhao QH, Jiang R, Wang L, Yuan P, Liu JM, Wu Y, Zhang J. The expression profiling of microRNA in systemic sclerosisassociated pulmonary arterial hypertension. Ann Transl Med 2021;9(18):1458. doi: 10.21037/atm-21-4342
38. Sarszegi Z, Szabo D, Gaszner B, et al. Examination of Pituitary Adenylate Cyclase-Activating Polypeptide (PACAP) as a Potential Biomarker in Heart Failure Patients. J Mol Neurosci 2019;68:368-76.

39. Hautmann M, Friis UG, Desch M, et al. Pituitary adenylate cyclase-activating polypeptide stimulates renin secretion via activation of PAC1 receptors. J Am Soc Nephrol 2007;18:1150-6.

(English Language Editor: J. Teoh) 